\title{
REFLECTION AT FREE SURFACE IN MICROPOLAR THERMOELASTIC SOLID WITH TWO TEMPERATURES
}

\author{
K. SHARMA \\ Department of Mechanical Engineering \\ Ecole Polytechnique Fédérale de Lausanne (EPFL) \\ Lausanne, SWITZERLAND \\ E-mail: kunal.sharma@epfl.ch
}

\begin{abstract}
The present investigation is concerned with the effect of two temperatures on reflection coefficients in a micropolar thermoelastic solid half space. With two relaxation times, reflection of plane waves impinging obliquely at a plane interface of the micropolar generalized thermoelastic solid half space with two temperatures is investigated. The incident wave is assumed to be striking at the plane surface after propagating through the micropolar generalized thermoelastic solid with two temperatures. Amplitude ratios of the various reflected waves are obtained in closed form and it is found that these are functions of angle of incidence, frequency and are affected by the elastic properties of the media. The effect of two temperatures is shown on these amplitude ratios for a specific model.
\end{abstract}

Key words: micropolar thermoelastic solid, conductive and thermodynamic temperatures, elastic waves, reflection coefficient.

\section{Introduction}

The theory of micropolar elasticity introduced and developed by Eringen (1966), has aroused much interest in recent years because of its possible utility in investigating the deformation properties of solids for which the classical theory is inadequate. The micropolar theory is believed to be particularly useful in investigating materials consisting of bar like molecules, which exhibit microrotational effects and can support body and surface couples. A micropolar continuum is a collection of interconnected particles in the form of small rigid bodies undergoing both translational and rotational motions. The force at a point of the surface element of bodies is completely characterized by the stress vector at that point.

The linear theory of micropolar thermoelasticity was developed by extending the theory of micropolar continua to include the thermal effect. The comprehensive review on the subject was given by Eringen $(1970 ; 1999)$ and Nowacki (1986). Touchert et al. (1968) also derived the basic equations of the linear theory of micropolar coupled thermoelasticity. Dost and Taborrok (1978) presented the generalized thermoelasticity by using Green and Lindsay theory. Chandrasekharaiah (1986) developed a heat flux dependent micropolar thermoelsticity. Boschi and Iesan (1973) extended a generalized theory of micropolar thermoelasticity that permits the transmission of heat as thermal waves at finite speed.

Thermoelasticity with two temperatures is one of the non-classical theories of thermoelasticity of elastic solids. The main difference of this theory with respect to the classical one is the thermal dependence. Chen et al. $(1968 ; 1969)$ formulated a theory of heat conduction in deformable bodies. Conduction depends on two distinct temperatures, the conductive temperature $\phi$ and thermodynamic temperature $\theta$. Chen et al (1969) suggested that the difference between these two temperatures is proportional to the heat supply. These two temperatures may be equal under certain conditions for time independent cases. However for time dependent problems, relating to wave propagation, these two temperatures are in general different, regardless of the presence of heat supply. The two temperatures and the strain are found to have representation in the form of a travelling wave pulse, a response which occurs instantaneously throughout the body (Boley, 1956). 
Warren and Chen (1973) investigated the wave propagation in the two temperature theory of thermoelasticity.

Youssef (2005) presented a new theory of generalized thermoelasticity by taking into account the theory of heat conduction in deformable bodies, which depends on distinct conductive and thermodynamics temperatures. He also established a uniqueness theorem for the equation of two temperature generalized linear thermoelasticity for a homogeneous and isotropic body. Different authors, Puri and Jordan (2006), Youssef and Al-Lehaibi (2007), Youssef and Al-Harby (2007), Magana and Quintanilla (2008), Mukhopadhyay and Kumar (2009), Roushan and Santwana (2010), Kaushal et al. (2010) and Kaushal et al. (2011) studied different problems in thermoelastic media with two temperatures.

Different authors (Parfitt and Eringen, 1969; Kumar and Singh, 1998a; Singh and Kumar, 1998b; Tomar et al., 1998; Kumar, 2000; Kumar and Sharma, 2005; Hsia and Cheng, 2006; Hsia et al., 2007; Singh, 2007; Kumar and Rupender, 2008; 2009) investigated the problems of reflection at the free surface of a micropolar elastic half space and micropolar thermoelastic half space.

In this paper, we study the problem of reflection of plane waves at free surface of a micropolar thermoelastic solid half space with two temperatures. The effect of two temperatures is depicted graphically on the amplitude ratios for incidence of various plane waves, i.e., Longitudinal displacement wave (LD wave), Thermal wave (T wave), Coupled transverse wave (CD-I wave and CD-II wave).

\section{Basic equations}

Following Ezzat and Aiwad (2010) and Green and Lindsay (1972), the field equations in an isotropic, homogeneous, micropolar elastic medium in the context of generalized theory of thermoelasticity with two temperatures, without body forces, body couples and heat sources, are given by

$$
\begin{aligned}
& (\lambda+2 \mu+K) \nabla(\nabla \cdot \boldsymbol{u})-(\mu+K) \nabla \times(\nabla \times \boldsymbol{u})+K(\nabla \times \phi)-v\left(1+\tau_{1} \frac{\partial}{\partial t}\right) \nabla T=\rho \frac{\partial^{2} \boldsymbol{u}}{\partial t^{2}}, \\
& (\alpha+\beta+\gamma) \nabla(\nabla \cdot \phi)-\gamma \nabla \times(\nabla \times \phi)+K(\nabla \times \boldsymbol{u})-2 K \phi=\rho \hat{j} \frac{\partial^{2} \phi}{\partial t^{2}} \\
& K^{*} \nabla^{2} \Phi=\rho c^{*}\left(\frac{\partial T}{\partial t}+\tau_{0} \frac{\partial^{2} T}{\partial t^{2}}\right)+v \Phi_{0} \frac{\partial}{\partial t}(\nabla \cdot \boldsymbol{u})
\end{aligned}
$$

where

$$
T=\left(1-a \nabla^{2}\right) \Phi
$$

and the constitutive relations are

$$
\begin{aligned}
& t_{i j}=\lambda u_{r, r} \delta_{i j}+\mu\left(u_{i, j}+u_{j, i}\right)+K\left(u_{j, i}+\varepsilon_{i j r} \phi_{r}\right)-v\left(1+\tau_{1} \frac{\partial}{\partial t}\right)\left(1-a \nabla^{2}\right) \Phi \delta_{i j}, \\
& m_{i j}=\alpha \phi_{r, r} \delta_{i j}+\beta \phi_{i, j}+\gamma \phi_{j, i}, \quad i, j, r=1,2,3
\end{aligned}
$$

where $\lambda$ and $\mu$ are Lame's constants. $K, \alpha, \beta$ and $\gamma$ are micropolar constants. $t_{i j}$ are the components of the stress tensor and $m_{i j}$ are the components of the couple stress tensor. $\boldsymbol{u}$ and $\phi$ are the displacement and microrotation vectors, $\delta_{i j}$ is the Kronecker delta, $\rho$ is the density, $\varepsilon_{i j r}$ is the alternating symbol, $\hat{j}$ is the 
microinertia, $K^{*}$ is the thermal conductivity, $c^{*}$ is the specific heat at constant strain, $T_{0}$ is the uniform temperature, $T$ is the temperature change, $v=(3 \lambda+2 \mu+K) \alpha_{T}$, where $\alpha_{T}$ is the coefficient of linear thermal expansion.

\section{Formulation of the problem}

We consider a homogeneous, isotropic, micropolar, thermoelastic solid half space with two temperatures. The rectangular Cartesian co-ordinate system $\mathrm{Ox}_{1} x_{2} x_{3}$ having origin on the surface $x_{3}=0$ with the $x_{3}$-axis pointing vertically into the solid medium is introduced.

We consider a two dimensional problem in the $x_{1} x_{3}$-plane, where the displacement vector $\boldsymbol{u}$ and microrotation vector $\phi$ for the solid medium $M$ are taken as

$$
\boldsymbol{u}=\left(u_{1}\left(x_{1}, x_{3}\right), 0, u_{3}\left(x_{1}, x_{3}\right)\right), \quad \phi=\left(0, \phi_{2}\left(x_{1}, x_{3}\right), 0\right) .
$$

For convenience, the following non dimensional quantities are introduced

$$
\begin{aligned}
& x_{1}^{\prime}=\frac{\omega^{*} x_{1}}{c_{1}}, \quad x_{3}^{\prime}=\frac{\omega^{*} x_{3}}{c_{1}}, \quad u_{1}^{\prime}=\frac{\rho \omega^{*} c_{1}}{v T_{0}} u_{1}, \quad u_{3}^{\prime}=\frac{\rho \omega^{*} c_{1}}{v T_{0}} u_{3}, \quad \phi_{2}^{\prime}=\frac{\rho c_{1}^{2}}{v T_{0}} \phi_{2}, \\
& t^{\prime}=\omega^{*} t, \quad \tau_{1}^{\prime}=\omega^{*} \tau_{1}, \quad T^{\prime}=\frac{T}{T_{0}}, \quad \Phi^{\prime}=\frac{\Phi}{\Phi_{0}}, \quad t_{i j}^{\prime}=\frac{1}{v T_{0}} t_{i j}, \quad m_{i j}^{\prime}=\frac{\omega^{*}}{c_{1} v T_{0}} m_{i j}, \\
& \tau_{0}^{\prime}=\omega^{*} \tau_{0}
\end{aligned}
$$

where

$$
\omega^{*}=\frac{\rho c^{*} c_{1}^{2}}{K^{*}}, \quad c_{1}^{2}=\frac{\lambda+2 \mu+K}{\rho} .
$$

The expressions relating the displacement components $u_{1}, u_{3}$ to the potential functions $\phi, \psi$ in dimensionless form are taken as

$$
u_{1}=\frac{\partial \phi}{\partial x_{1}}-\frac{\partial \psi}{\partial x_{3}}, \quad u_{3}=\frac{\partial \phi}{\partial x_{3}}+\frac{\partial \psi}{\partial x_{1}}
$$

Making use of Eqs (3.3) in Eqs (2.1)-(2.3) and with the aid of Eqs (3.1) and (3.2); (after suppressing the primes), we obtain

$$
\begin{aligned}
& \nabla^{2} \phi-\left(1-a \nabla^{2}\right) \Phi-\frac{\partial^{2} \phi}{\partial t^{2}}=0, \\
& \nabla^{2} \psi-a_{1} \phi_{2}-a_{2} \frac{\partial^{2} \psi}{\partial t^{2}}=0,
\end{aligned}
$$




$$
\begin{aligned}
& \nabla^{2} \phi_{2}-a_{3} \nabla^{2} \psi-a_{4} \phi_{2}-a_{5} \frac{\partial^{2} \phi_{2}}{\partial t^{2}}=0 \\
& \nabla^{2} \Phi=a_{6}\left(1+\tau_{0} \frac{\partial}{\partial t}\left(1-a \nabla^{2}\right) \Phi\right) \frac{\partial}{\partial t}+a_{7} \frac{\partial}{\partial t} \nabla^{2} \phi
\end{aligned}
$$

where

$$
\begin{aligned}
& a_{1}=\frac{K}{\mu+K}, \quad a_{2}=\frac{\rho c_{1}^{2}}{\mu+K}, \quad a_{3}=\frac{K c_{1}^{2}}{\gamma \omega^{* 2}}, \quad a_{4}=2 a_{3}, \quad a_{5}=\frac{\rho \hat{j} c_{1}^{2}}{\gamma}, \\
& a_{6}=\frac{\rho c^{*} c_{1}^{2} T_{0}}{K^{*} \omega^{*} \Phi_{0}}, \quad a_{7}=\frac{v c_{1}^{2}}{K^{*} \omega^{*}},
\end{aligned}
$$

and $\nabla^{2}=\frac{\partial^{2}}{\partial x_{1}^{2}}+\frac{\partial^{2}}{\partial x_{3}^{2}}$ is the Laplacian operator.

\section{Boundary conditions}

The boundary conditions at the free surface $x_{3}=0$ are such that normal stress, tangential stress, tangential couple stress and temperature gradient vanish. Mathematically, these can be written as

$$
T_{33}=0, \quad T_{31}=0, \quad m_{32}=0, \quad \frac{\partial T}{\partial x_{3}}=0 .
$$

\section{Reflection}

We consider the Longitudinal displacement wave (LD-wave), Thermal wave (T-wave), Coupled transverse and microrotational waves (CD-I wave and CD-II wave) propagating through the medium $M$ and incident at the plane $x_{3}=0$ with its direction of propagation with angle $\theta_{0}$ normal to the surface. Corresponding to each incident wave, we get reflected LD-wave, T-wave, CD-I and CD-II waves in medium $M$ as shown in Fig.1.

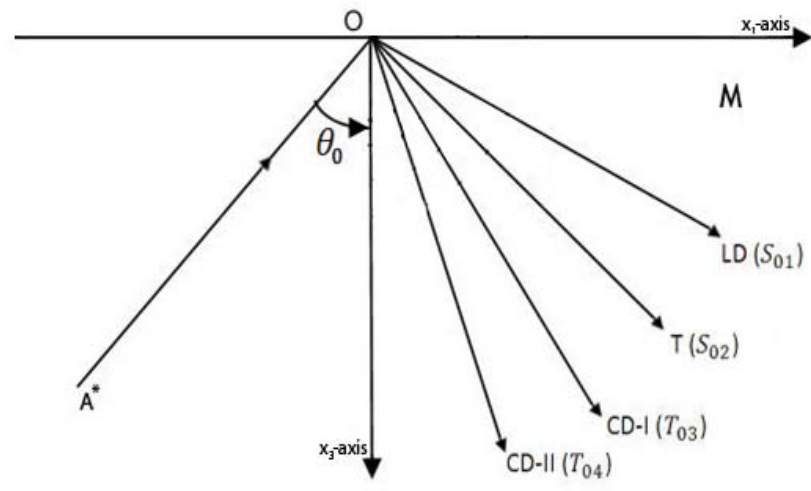

Fig.1. Geometry of the problem.

In order to solve Eqs (3.4)-(3.7), we assume the solutions of the form 


$$
\left\{\phi, T, \Phi, \psi, \phi_{2}\right\}=\left\{\tilde{\phi}, \tilde{T}, \tilde{\Phi}, \tilde{\psi}, \tilde{\phi}_{2}\right\} e^{\left\{\left\{k\left(x_{1} \sin \theta-x_{3} \cos \theta\right)-\omega t\right\}\right.}
$$

where $k$ is the wave number and $\omega$ is the angular frequency and $\phi, T, \Phi, \psi, \phi_{2}$ are arbitrary constants.

The use of Eq.(5.1) in Eqs (3.4)-(3.7), yield

$$
\begin{aligned}
& V^{4}+D_{1} V^{2}+E_{1}=0, \\
& V^{4}+D_{2} V^{2}+E_{2}=0
\end{aligned}
$$

where

$$
\begin{aligned}
& D_{1}=\left(\frac{a_{1} a_{3}}{\omega^{2} a_{2}}-1\right) \frac{1}{\left(a_{5}-\frac{a_{4}}{\omega^{2}}\right)}-\frac{1}{a_{2}}, \quad E_{1}=\frac{1}{\left(a_{5}-\frac{a_{4}}{\omega^{2}}\right) a_{2}}, \\
& D_{2}=\frac{1+\left(a-\frac{1}{\omega^{2}}\right) a_{6} \omega^{4}\left(\frac{1}{\omega}+\tau_{0}\right)+a_{7} \omega^{2}\left(\frac{1}{\omega}+\tau_{0}\right)}{\omega^{2} a_{6}\left(\frac{1}{\omega}+\tau_{0}\right)-1}, E_{2}=\frac{a \omega^{2}\left[a_{7}\left(\frac{1}{\omega}+\tau_{0}\right)-a_{6}\left(\frac{1}{\omega}+\tau_{0}\right)\right]}{a_{6}\left(\frac{1}{\omega}+\tau_{0}\right)-\frac{1}{\omega^{2}}} .
\end{aligned}
$$

Equations (5.2) and (5.3) are quadratic in $V^{2}$, therefore the roots of these equations give four values of $V^{2}$. Corresponding to each value of $V^{2}$ in Eq.(5.2), there exist two types of waves in the solid medium in a decreasing order of their velocities, namely an LD-wave, T-wave. Similarly, corresponding to each value of $V^{2}$ in Eq.(5.3), there exist two types of waves in the solid medium, namely a CD-I wave, CD-II wave. Let $V_{1}, V_{2}$ be the velocities of the reflected LD-wave, T-wave and $V_{3}, V_{4}$ the velocities of the reflected CD-I wave, CD-II wave in medium $M$.

In view of Eq.(5.1), the appropriate solutions of Eqs (3.4)-(3.7) for medium $M$ is assumed in the form

$$
\begin{aligned}
& \{\phi, \Phi\}=\sum_{i=1}^{2}\left\{1, f_{i}\right\}\left[S_{0 i} e^{\left\{\left\{k_{i}\left(x_{1} \sin \theta_{0 i}-x_{3} \cos \theta_{0 i}\right)-\omega_{i} t\right\}\right.}+P_{i}\right] \\
& \left\{\psi, \phi_{2}\right\}=\sum_{j=3}^{4}\left\{1, f_{i}\right\}\left[T_{0 j} e^{1\left\{k_{j}\left(x_{1} \sin \theta_{0 j}-x_{3} \cos \theta_{0 j}\right)-\omega_{j} t\right\}}+P_{j}\right]
\end{aligned}
$$

where

$$
P_{i}=S_{i} e^{\left\{\left\{k_{i}\left(x_{I} \sin \theta_{0 i}+x_{3} \cos \theta_{0 i}\right)-\omega_{i} t\right\}\right.}, \quad P_{j}=T_{j} e^{\left\{\left\{k_{j}\left(x_{1} \sin \theta_{0 j}+x_{3} \cos \theta_{0 j}\right)-\omega_{j} t\right\}\right.},
$$

and $S_{0 i}, T_{0 j}$ are the amplitudes of incident (LD-wave, T-wave) and (CD-I, CD-II) waves respectively. $S_{i}$ and $T_{j}$ are the amplitudes of the reflected (LD-wave, T-wave) and (CD-I, CD-II) waves.

In order to satisfy the boundary conditions, we use the following extension of Snell's law

$$
\frac{\sin \theta_{0}}{V_{0}}=\frac{\sin \theta_{1}}{V_{1}}=\frac{\sin \theta_{2}}{V_{2}}=\frac{\sin \theta_{3}}{V_{3}}=\frac{\sin \theta_{4}}{V_{4}}
$$

where

$$
k_{1} V_{1}=k_{2} V_{2}=k_{3} V_{3}=k_{4} V_{4}=\omega, \quad \text { at } \quad x_{3}=0 \text {. }
$$


Making use of the values of $\phi, \psi, \Phi$ and $\phi_{2}$ from Eqs (5.4) and (5.5) in the boundary conditions (4.1) and with the aid of Eqs (2.4)-(3.3) and using Eqs (5.6) and (5.7), we obtain a system of four nonhomogeneous equations which can be written as

$$
\sum_{j=1}^{4} a_{i j} Z_{j}=Y_{i} ; \quad(i=1,2,3,4)
$$

where

$$
\begin{aligned}
& a_{1 i}=\left(d_{1}+d_{2}\left(1-\frac{V_{i}^{2}}{V_{0}^{2}} \sin ^{2} \theta_{0}\right)\right) \frac{V_{i}^{2}}{\omega^{2}}+\left(1-\tau_{1} 1 \omega\right)\left(1+a \frac{V_{i}^{2}}{\omega^{2}}\right) f_{i}, \\
& a_{1 j}=\frac{V_{j}^{3}}{\omega^{2} V_{0}} \sin \theta_{0} \sqrt{1-\frac{V_{j}^{2}}{V_{0}^{2}} \sin ^{2} \theta_{0}}, \quad a_{2 i}=-\left(2 d_{4}+d_{5}\right) \frac{V_{i}^{3}}{\omega^{2} V_{0}} \sin \theta_{0} \sqrt{1-\frac{V_{i}^{2}}{V_{0}^{2}} \sin ^{2} \theta_{0}}, \\
& a_{2 j}=\left(2 d_{4} \frac{V_{j}^{2}}{\omega^{2}}\left(1-\frac{V_{j}^{2}}{V_{0}^{2}} \sin ^{2} \theta_{0}\right)-d_{5} \frac{V_{j}^{4}}{\omega^{2} V_{0}^{2}} \sin ^{2} \theta_{0}\right)-d_{5} f_{j} \\
& a_{3 i}=0, \quad a_{3 j}=\frac{V_{j}}{\omega} \sqrt{1-\frac{V_{j}^{2}}{V_{0}^{2}} \sin ^{2} \theta_{0}} f_{j}, \quad a_{4 i}=\imath \frac{V_{i}}{\omega}\left(1+a \frac{V_{i}^{2}}{\omega^{2}}\right) \sqrt{1-\frac{V_{i}^{2}}{V_{0}^{2}} \sin ^{2} \theta_{0}} f_{i}, \quad a_{4 j}=0, \\
& (i=1,2 \text { and } j=3,4)
\end{aligned}
$$

and

$$
Z_{1}=\frac{S_{1}}{A^{*}}, \quad Z_{2}=\frac{S_{2}}{A^{*}}, \quad Z_{3}=\frac{T_{3}}{A^{*}}, \quad Z_{4}=\frac{T_{4}}{A^{*}},
$$

where $Z_{1}, Z_{2}, Z_{3}, Z_{4}$ are the complex amplitude ratios of the reflected LD-wave, T-wave and coupled CD-I, CD-II waves in medium $M$.

The phase of the reflected waves for the incident LD-wave, T-wave and CD-I waves can be written by using Eqs (5.6) and (5.7) as

$$
\frac{\cos \theta_{i}}{V_{i}}=\frac{1}{V_{0}}\left[\left(\frac{V_{0}}{V_{i}}\right)^{2}-\sin ^{2} \theta_{0}\right]^{1 / 2}, \quad \frac{\cos \theta_{j}}{V_{j}}=\frac{1}{V_{0}}\left[\left(\frac{V_{0}}{V_{j}}\right)^{2}-\sin ^{2} \theta_{0}\right]^{1 / 2} .
$$

Following Schoenberg (1971), we can write

$$
\begin{aligned}
& \frac{\cos \theta_{i}}{V_{i}}=\frac{\cos \theta_{i}^{\prime}}{V_{i}^{\prime}}+\frac{c_{i}}{V_{0} 2 \pi}, \quad(i=1,2,3,4,5) \text { then } \\
& \frac{\cos \theta_{i}^{\prime}}{V_{i}^{\prime}}=\frac{1}{V_{0}} \operatorname{Re}\left\{\left[\left(\frac{V_{0}}{V_{i}}\right)^{2}-\sin ^{2} \theta_{0}\right]^{1 / 2}\right\}, \quad c_{i}=2 \pi \operatorname{Im}\left\{\left[\left(\frac{V_{0}}{V_{i}}\right)^{2}-\sin ^{2} \theta_{0}\right]^{1 / 2}\right\}
\end{aligned}
$$


where $V_{i}^{\prime}$, the real phase speed and $\theta_{i}^{\prime}$, the angle of reflection are given by

$$
\frac{V_{i}^{\prime}}{V_{0}}=\frac{\sin \theta_{i}^{\prime}}{\sin \theta_{0}}\left[\sin ^{2} \theta_{0}+\left[\operatorname{Re}\left[\left(\frac{V_{0}}{V_{i}}\right)^{2}-\sin ^{2} \theta_{0}\right]^{1 / 2}\right]^{2}\right]^{-1 / 2},
$$

and $c_{i}$, the attenuation in a depth is equal to the wavelength of incident wave, i.e., $2 \pi \frac{V_{0}}{\omega}$ and here Re and Im denote Real and Imaginary parts.

(1) For the incident LD-wave

$$
\begin{aligned}
& A^{*}=S_{01}, \quad S_{02}=T_{03}=T_{04}=0, \quad Y_{1}=-a_{11}, \quad Y_{2}=a_{21}, \quad Y_{3}=a_{31}=0, \\
& Y_{4}=a_{41}, \quad Y_{5}=-a_{51}, \quad Y_{6}=a_{61}, \quad Y_{7}=a_{71}=0, \quad Y_{8}=-a_{81} .
\end{aligned}
$$

(2) For the incident T-wave

$$
\begin{aligned}
& A^{*}=S_{02}, \quad S_{01}=T_{03}=T_{04}=0, \quad Y_{1}=-a_{12}, \quad Y_{2}=a_{22}, \quad Y_{3}=a_{32}=0, \\
& Y_{4}=a_{42}, \quad Y_{5}=-a_{52}, \quad Y_{6}=a_{62}, \quad Y_{7}=a_{72}=0, \quad Y_{8}=-a_{82} .
\end{aligned}
$$

(3) For the incident CD-I wave

$$
\begin{aligned}
& A^{*}=T_{03}, \quad S_{01}=S_{02}=T_{04}=0, \quad Y_{1}=a_{13}, \quad Y_{2}=-a_{23}, \quad Y_{3}=a_{33}, \\
& Y_{4}=a_{43}=0, \quad Y_{5}=a_{53}, \quad Y_{6}=-a_{63}, \quad Y_{7}=-a_{73}, \quad Y_{8}=a_{83}=0 .
\end{aligned}
$$

(4) For the incident CD-II wave

$$
\begin{aligned}
& A^{*}=T_{04}, \quad S_{01}=S_{02}=T_{03}=0, \quad Y_{1}=a_{14}, \quad Y_{2}=-a_{24}, \quad Y_{3}=a_{34}, \\
& Y_{4}=a_{44}=0, \quad Y_{5}=a_{54}, \quad Y_{6}=-a_{64}, \quad Y_{7}=-a_{74}, \quad Y_{8}=a_{84}=0 .
\end{aligned}
$$

\section{Particular case}

If two temperature parameters vanish, i.e., $a=0$, then we obtain the amplitude ratios in the micropolar thermoelastic solid half space, where the values of $a_{i j}$ are given by

$$
\begin{aligned}
& a_{1 i}=\left(d_{1}+d_{2}\left(1-\frac{V_{i}^{2}}{V_{0}^{2}} \sin ^{2} \theta_{0}\right)\right) \frac{V_{i}^{2}}{\omega^{2}}+\left(1-\tau_{1} 1 \omega\right) f_{i}, \\
& a_{1 j}=\frac{V_{j}^{3}}{\omega^{2} V_{0}} \sin \theta_{0} \sqrt{1-\frac{V_{j}^{2}}{V_{0}^{2}} \sin ^{2} \theta_{0}}, \quad a_{2 i}=-\left(2 d_{4}+d_{5}\right) \frac{V_{i}^{3}}{\omega^{2} V_{0}} \sin \theta_{0} \sqrt{1-\frac{V_{i}^{2}}{V_{0}^{2}} \sin ^{2} \theta_{0}},
\end{aligned}
$$




$$
\begin{aligned}
& a_{2 j}=\left(2 d_{4} \frac{V_{j}^{2}}{\omega^{2}}\left(1-\frac{V_{j}^{2}}{V_{0}^{2}} \sin ^{2} \theta_{0}\right)-\left(d_{5} \frac{V_{j}^{2}}{\omega^{2} V_{0}^{2}} \sin ^{2} \theta_{0}\right)\right)-d_{5} f_{j}, \\
& a_{3 i}=0, \quad a_{3 j}=1 \frac{V_{j}}{\omega} \sqrt{1-\frac{V_{j}^{2}}{V_{0}^{2}} \sin ^{2} \theta_{0}} f_{j}, \quad a_{4 i}=1 \frac{V_{i}}{\omega} \sqrt{1-\frac{V_{i}^{2}}{V_{0}^{2}} \sin ^{2} \theta_{0}} f_{i}, \quad a_{4 j}=0, \\
& (i=1,2 \text { and } j=3,4) \text {. }
\end{aligned}
$$

\section{Numerical results and discussion}

For numerical computations, we take the following values of relevant parameters for both the half spaces. Following Eringen (1984), the values of micropolar constants for medium $M$ are taken as

$$
\begin{aligned}
& \lambda=9.4 \times 10^{10} \mathrm{Nm}^{-2}, \quad \mu=4.0 \times 10^{10} \mathrm{Nm}^{-2}, \quad \kappa=1.0 \times 10^{10} \mathrm{Nm}^{-2}, \\
& \gamma=7.79 \times 10^{-10} \mathrm{~N}, \quad \hat{j}=0.002 \times 10^{-17} \mathrm{~m}^{2}, \quad \rho=1.74 \times 10^{3} \mathrm{Kg} \mathrm{m}^{-3},
\end{aligned}
$$

and thermal parameters for medium $M$ are taken as

$$
\begin{aligned}
& T_{0}=0.298 K, \quad \Phi_{0}=0.292 \mathrm{~K}, \quad v=0.268 \times 10^{5} \mathrm{Nm}^{-2} \mathrm{~K}^{-1}, \\
& c^{*}=0.104 \times 10^{3} \mathrm{Jkg}^{-1} \mathrm{~K}^{-1}, \quad a=0.03 \mathrm{~m}^{2}, \quad K^{*}=1.7 \times 10^{2} \mathrm{~J} \mathrm{~m}^{-1} \mathrm{~s}^{-1} \mathrm{~K}^{-1}, \\
& \omega=1, \quad \tau_{0}=0.4 \mathrm{sec}, \quad \tau_{1}=0.2 \mathrm{sec} .
\end{aligned}
$$

In Figs 2-25, we use a solid line for the incident wave in the micropolar generalized thermoelastic solid $(a=0)$, small dashes line for the incident wave in the micropolar generalized thermoelastic solid with two temperatures $(a=0.3)$ and large dashes line for the incident wave in the micropolar generalized thermoelastic solid with two temperatures $(a=0.9)$.

\subsection{Incident LD-Wave}

Variations of amplitude ratios $\left|Z_{i}\right| ; 1 \leq i \leq 4$, with the angle of incidence $\theta_{0}$, for the incident LDwave are shown in Figs 2 through 5.

Figure 2 shows that the values of the amplitude ratio $\left|Z_{1}\right|$ for all the values of $a$ decrease in the whole range, except for $\theta_{0}>84^{\circ}$ where the values for $a=0$ and $a=0.3$ increase. Also the values for $a=0.9$ remain more than the values for $a=0.3$ and $a=0$ in the whole range except in the initial range where the values are same.

Figure 3 shows that the values of $\left|Z_{2}\right|$ for all the values of $a$ increase in the whole range except near $\theta_{0}=90^{\circ}$, where the values of the amplitude ratio decrease sharply. Also, the values for all three values of $a$ remain same with a slight difference in their magnitude.

Figure 4 shows that the values of $\left|Z_{3}\right|$ for all the values of $a$ first increase in the range $0^{\circ}<\theta_{0}<75^{\circ}$ and then decrease for subsequent values. Also, the values for $a=0$ remain more than the values for $a=0.3$ which remain more than the values for $a=0.9$. It is noted from Fig.5 that the behavior of variation of $\left|Z_{4}\right|$ for all the values of $a$ is similar. The values of $\left|Z_{4}\right|$ for $a=0.9$ remain greater than the values for $a=0.3$ and $a=0$. 


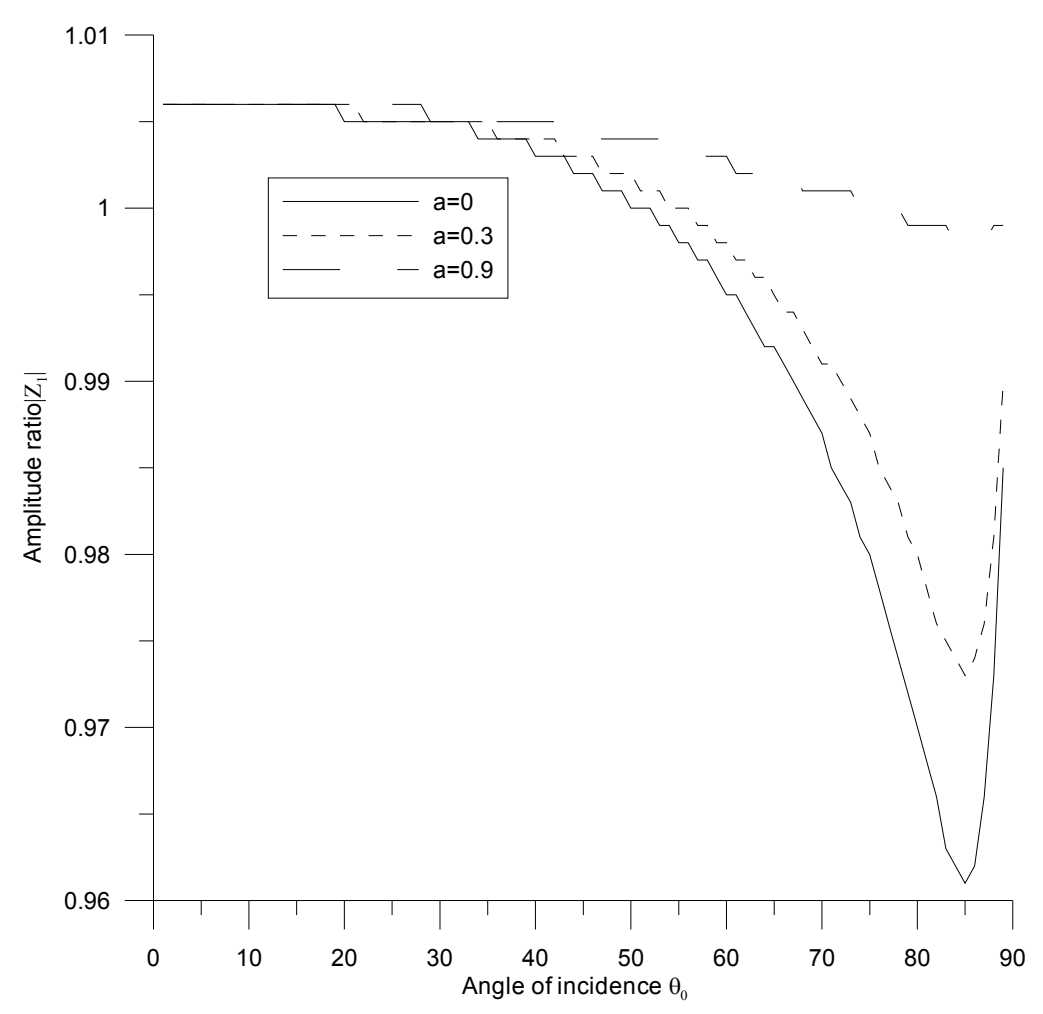

Fig.2.

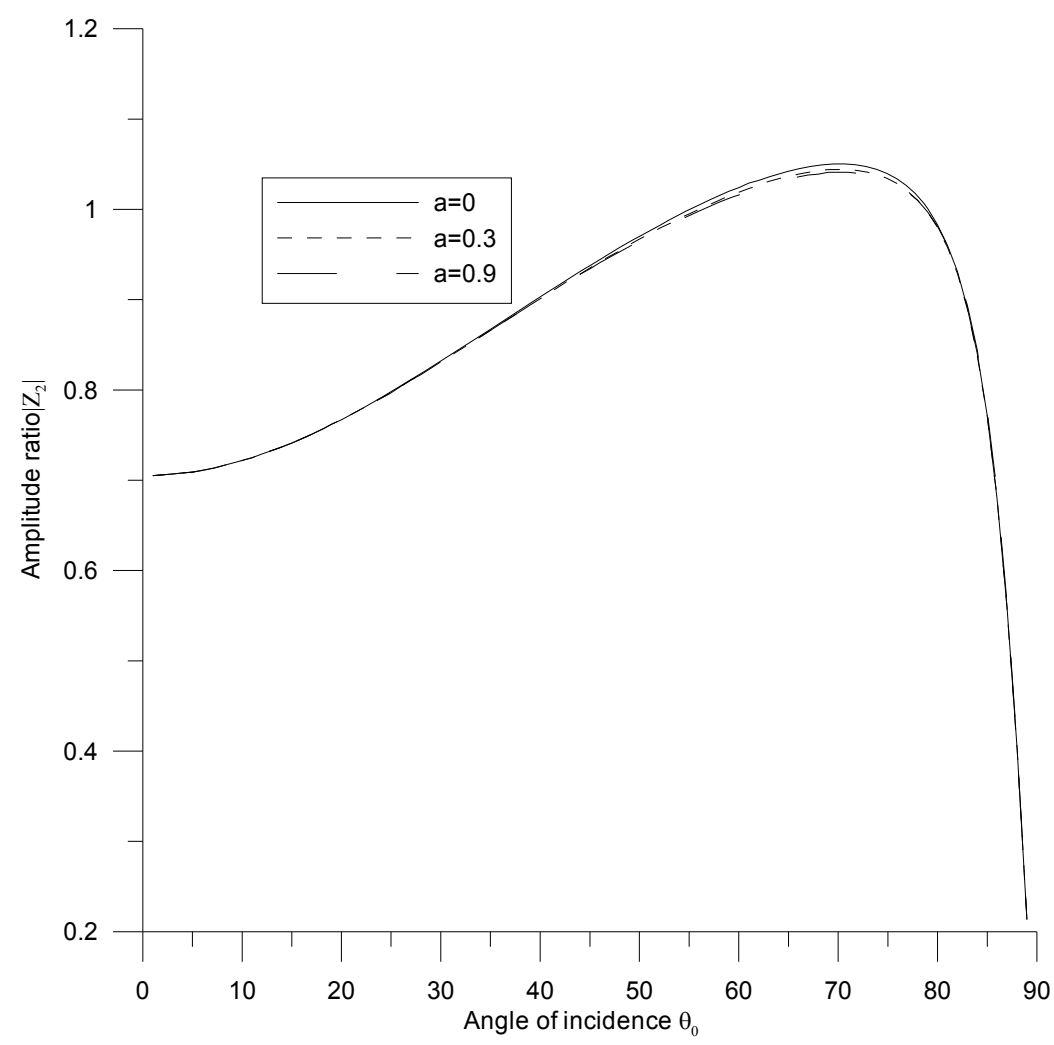

Fig.3. 


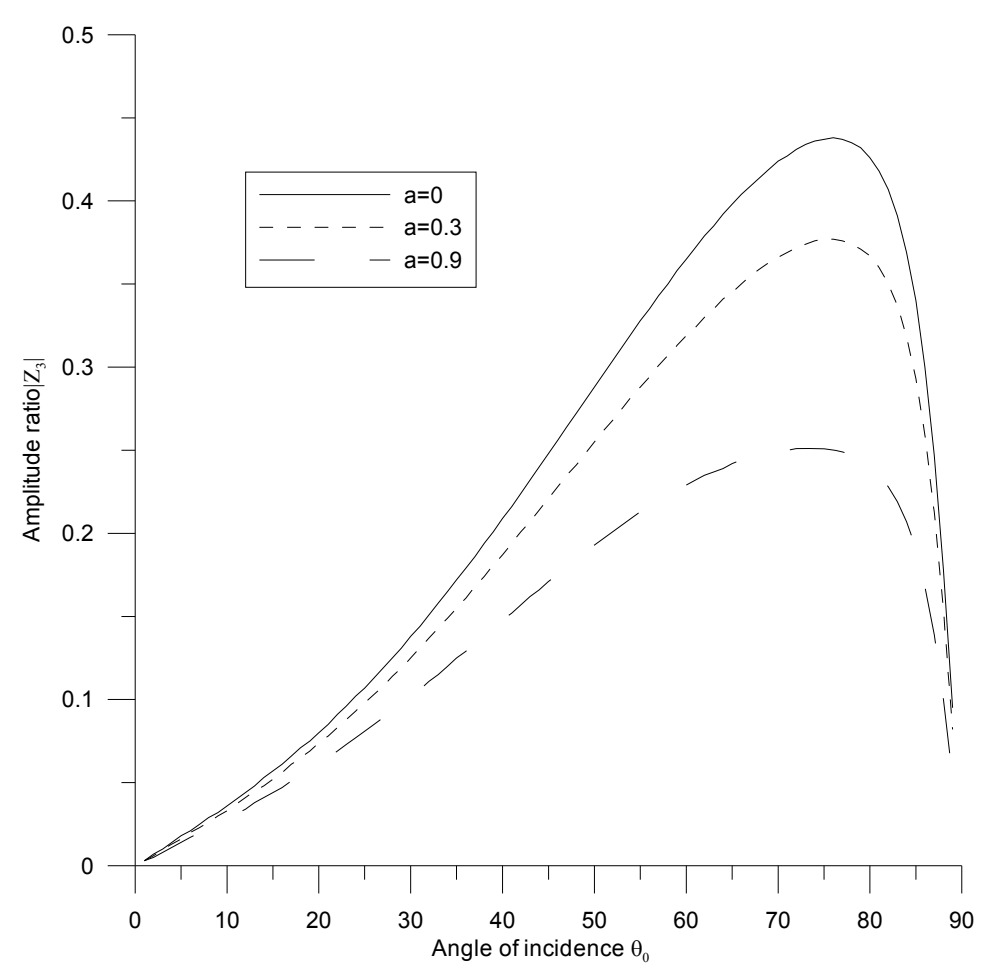

Fig.4.

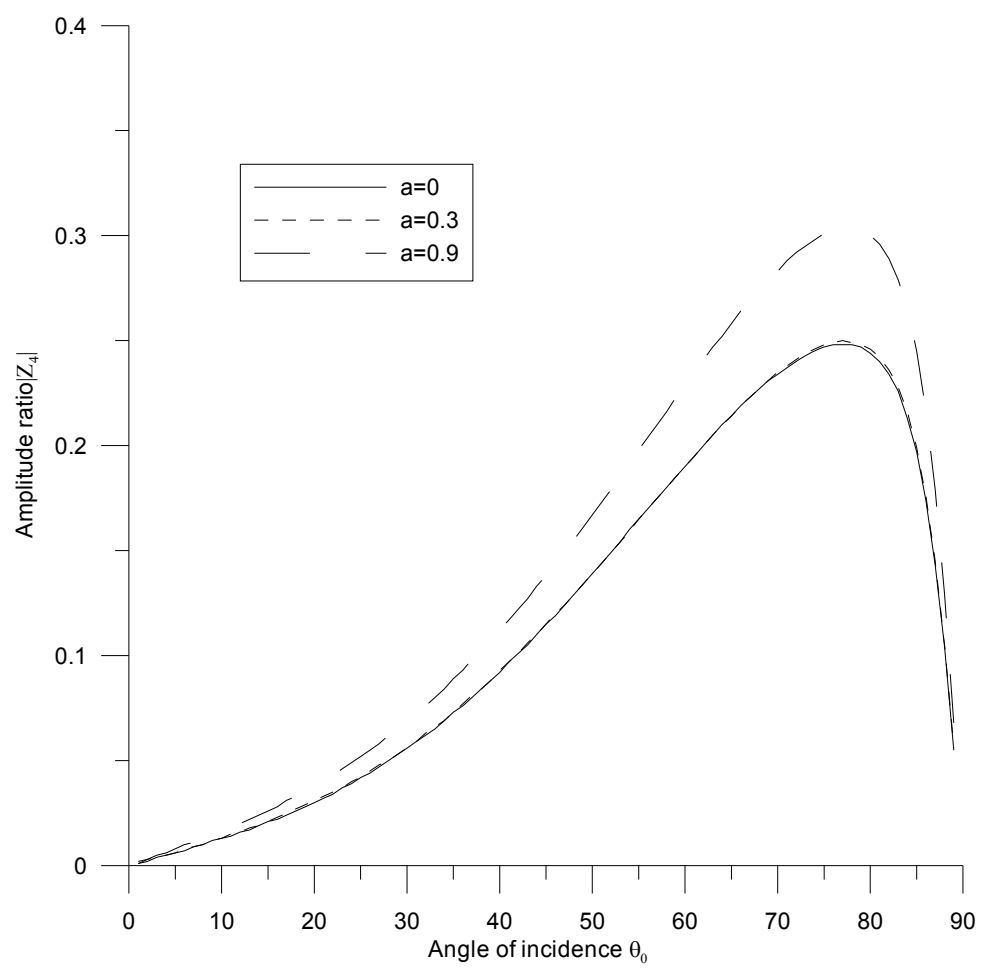

Fig.5.

Figs 2-5. Variations of amplitude ratios with the angle of incidence for LD-Wave. 


\subsection{Incident $\mathbf{T}$-Wave}

Variations of amplitude ratios $\left|Z_{i}\right| ; 1 \leq i \leq 4$, with the angle of incidence $\theta_{0}$, for the incident T- wave are shown in Figs 6 through 9.

Figure 6 illustrates that the values of $\left|Z_{l}\right|$ for all the values of $a$ decrease in the whole range and the values of the amplitude ratio for all the values of $a$ remain same in the whole range.

It is evident from Fig.7 that the values of $\left|Z_{2}\right|$ for all the values of $a$ first decrease in the initial range and then show an increase near $\theta_{0}=90^{\circ}$. Also, the values of the amplitude ratio decrease with a decrease in the value of $a$, that shows the effect of two temperatures.

Figure 8 shows that the behavior of variation of $\left|Z_{3}\right|$ for all the values of $a$ is similar with a difference in their magnitude. The values of $\left|Z_{3}\right|$ decrease with an increase in the value of $a$.

Figure 9 shows that the values of the amplitude ratio $\left|Z_{4}\right|$ decrease with a decrease in the value of $a$. The maximum value is attained for $a=0.9$ in the intermediate range.

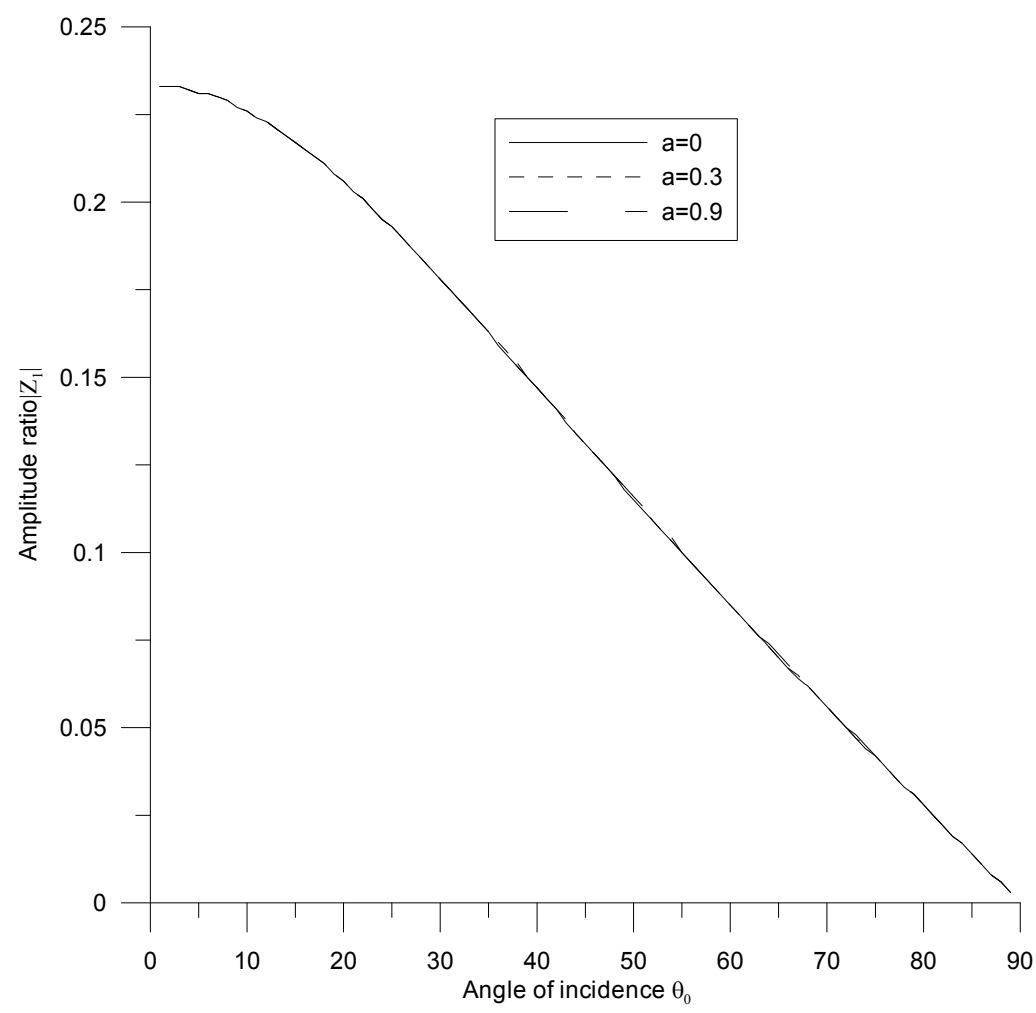

Fig.6. 


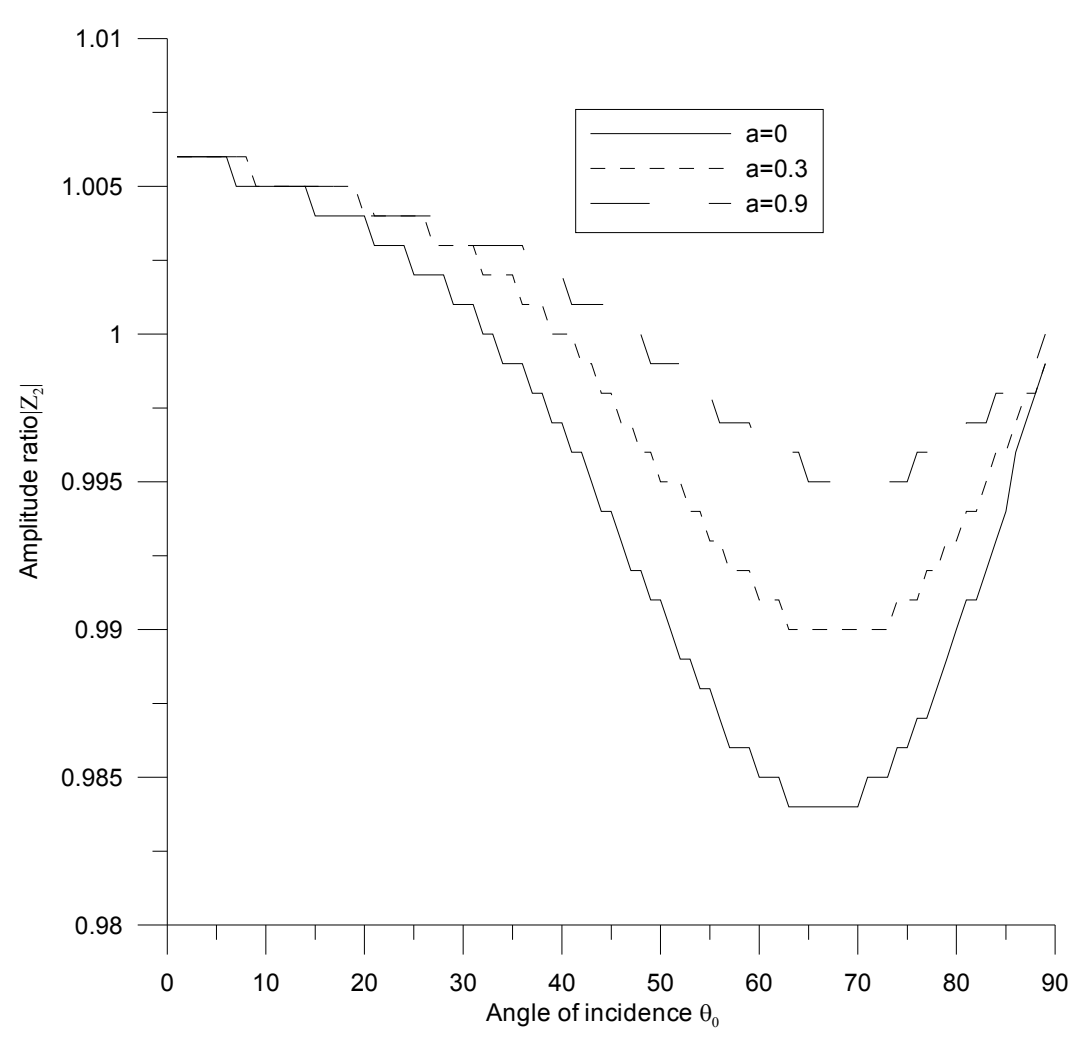

Fig.7.

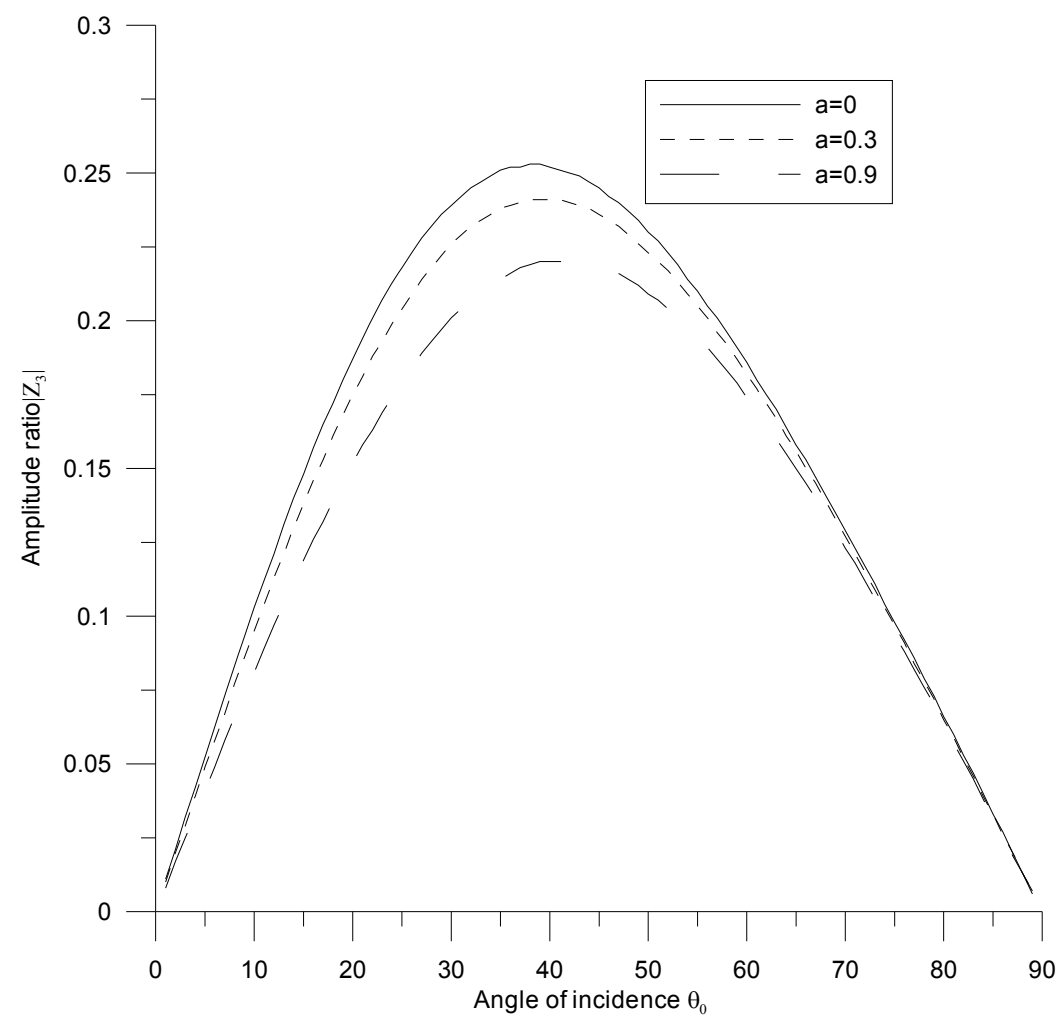

Fig. 8. 


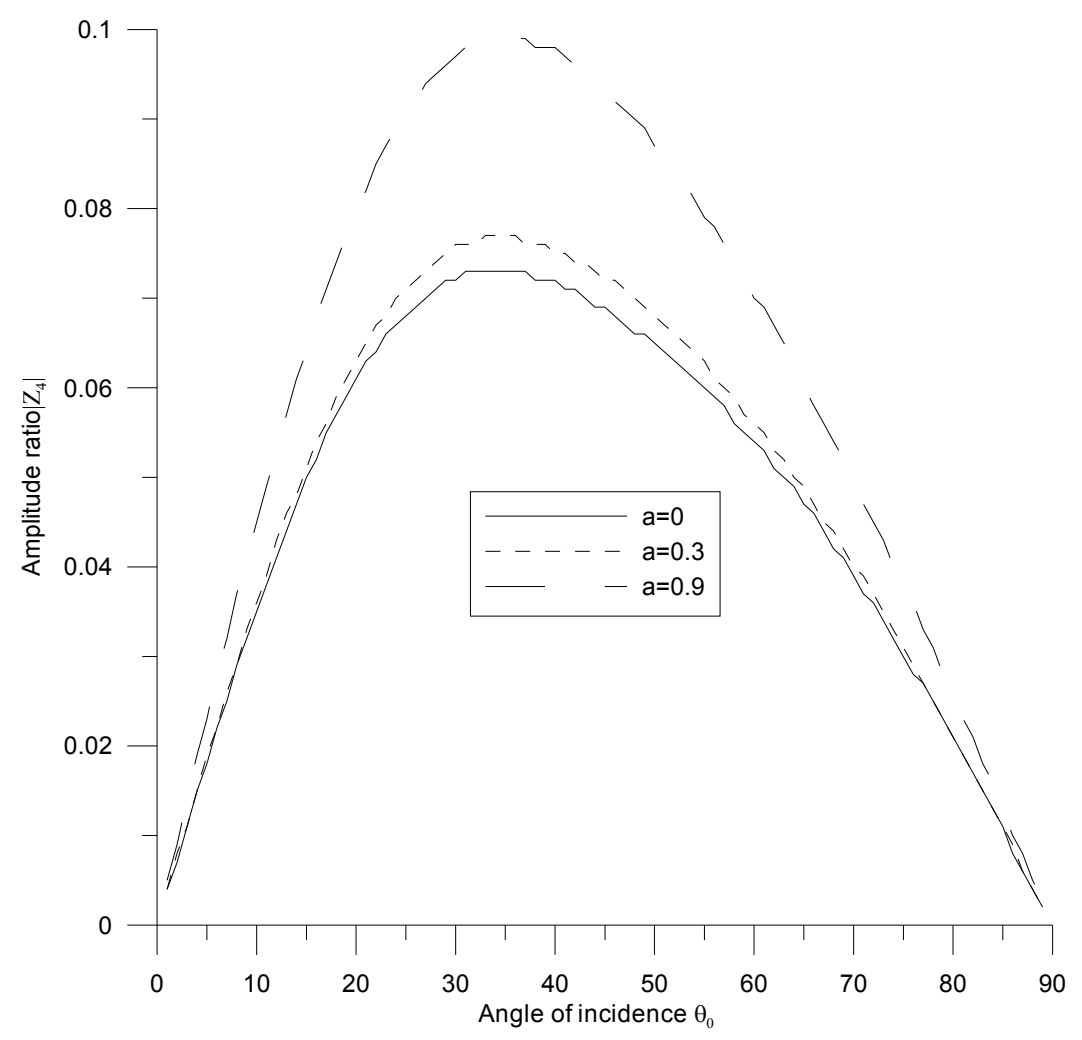

Fig.9.

Figs 6-9. Variations of amplitude ratios with the angle of incidence for T-Wave.

\subsection{Incident CD-I Wave}

Variations of amplitude ratios $\left|Z_{i}\right| ; 1 \leq i \leq 4$, with the angle of incidence $\theta_{0}$, for the incident CD-I wave are shown in Figs 10 through 13.

Figures 10 and 11 shows that the values of the amplitude ratio $\left|Z_{1}\right|$ and $\left|Z_{2}\right|$ for all the values of $a$ increase from normal incidence to intermediate range and then decrease from intermediate range to grazing incidence. Also from normal incidence to intermediate range the values for $a=0$ are more than the values for $a=0.3$ and $a=0.9$, while in the next range the values are similar.

It is noted from Fig. 12 that the values of $\left|Z_{3}\right|$ for all the values of $a$ increase in the whole range and the values for $a=0$ remain more than the values for $a=0.3$, which are greater than the values for $a=0.9$ in the whole range.

Figure 13 shows that the values of $\left|Z_{4}\right|$ for all the values of $a$ decrease in the whole range with a difference in their magnitude. Also, the values of the amplitude ratio decrease with a decrease in the value of $a$. 


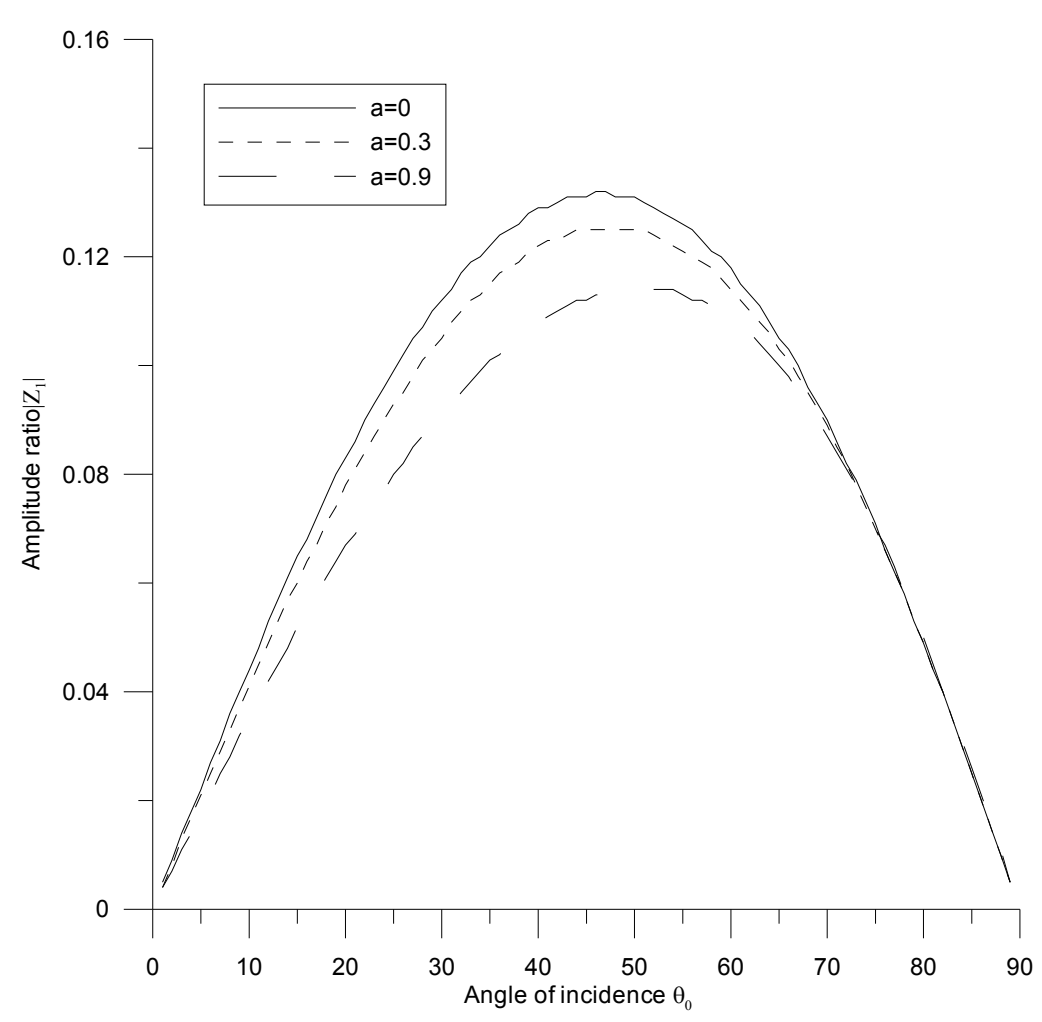

Fig.10.

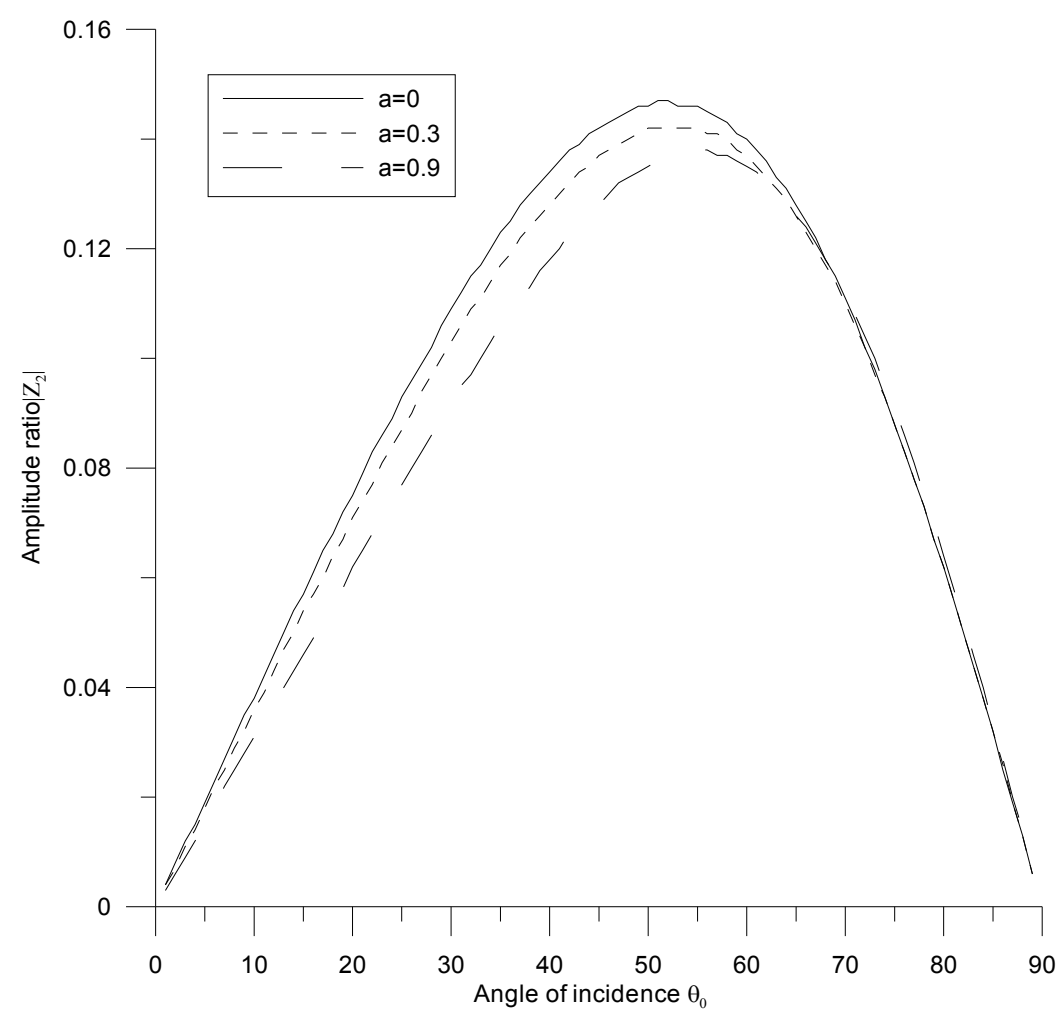

Fig.11. 


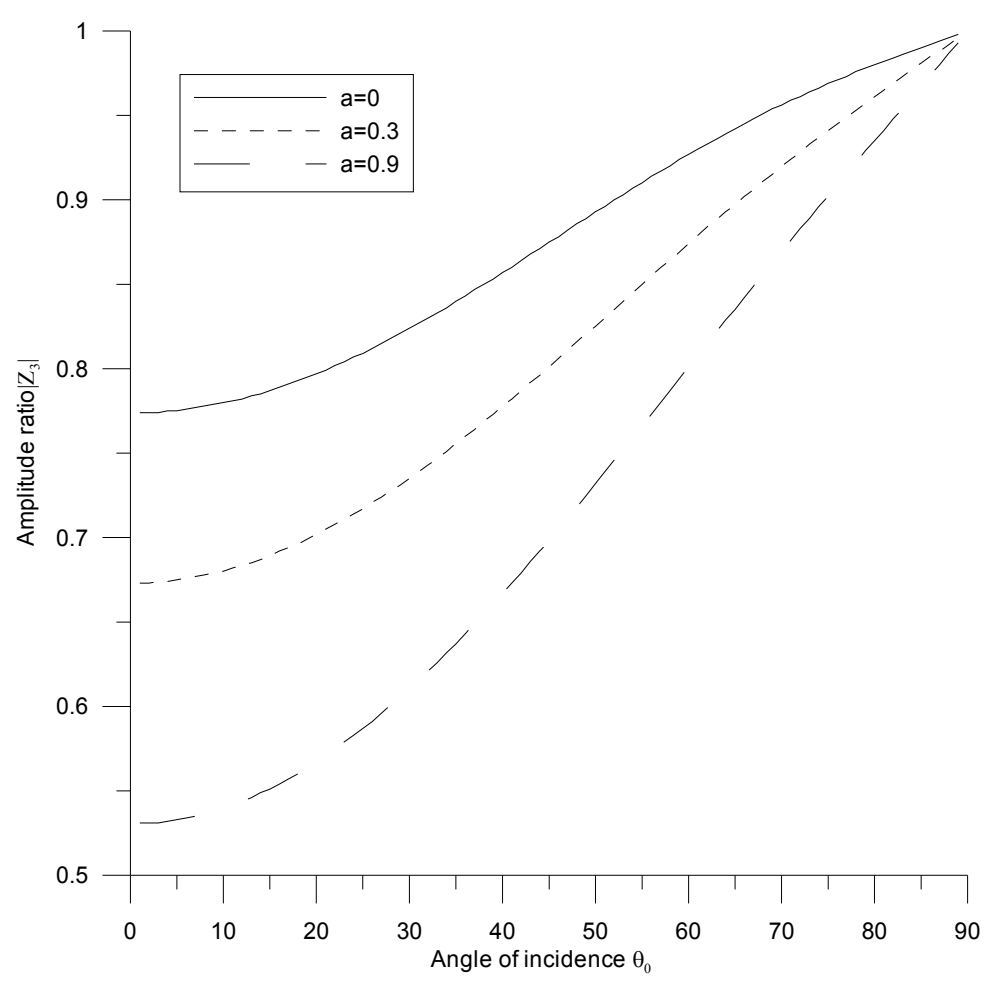

Fig.12.

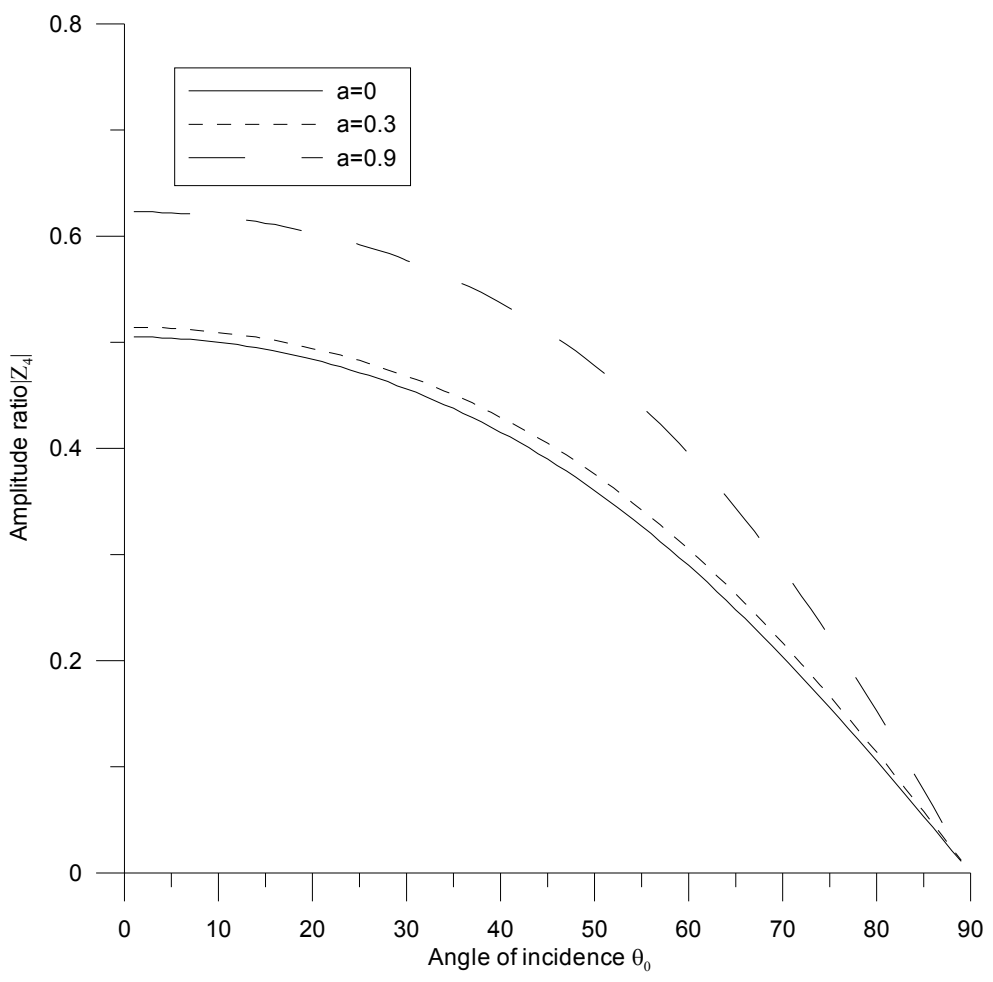

Fig.13.

Figs 10-13. Variations of amplitude ratios with the angle of incidence for CD-I Wave. 


\section{Conclusion}

An analytical expression for reflection coefficients of various reflected waves have been derived. It is observed that the values of $\left|Z_{3}\right|$ decrease with an increase in the value of $a$, for the incidence of all the plane waves that reveals the effect of two temperatures. Also, the values of $\left|Z_{4}\right|$ increase with an increase in the value of $a$, for the incidence of all the plane waves. These two temperatures have a significant impact on the amplitude ratios.

\section{Nomenclature}

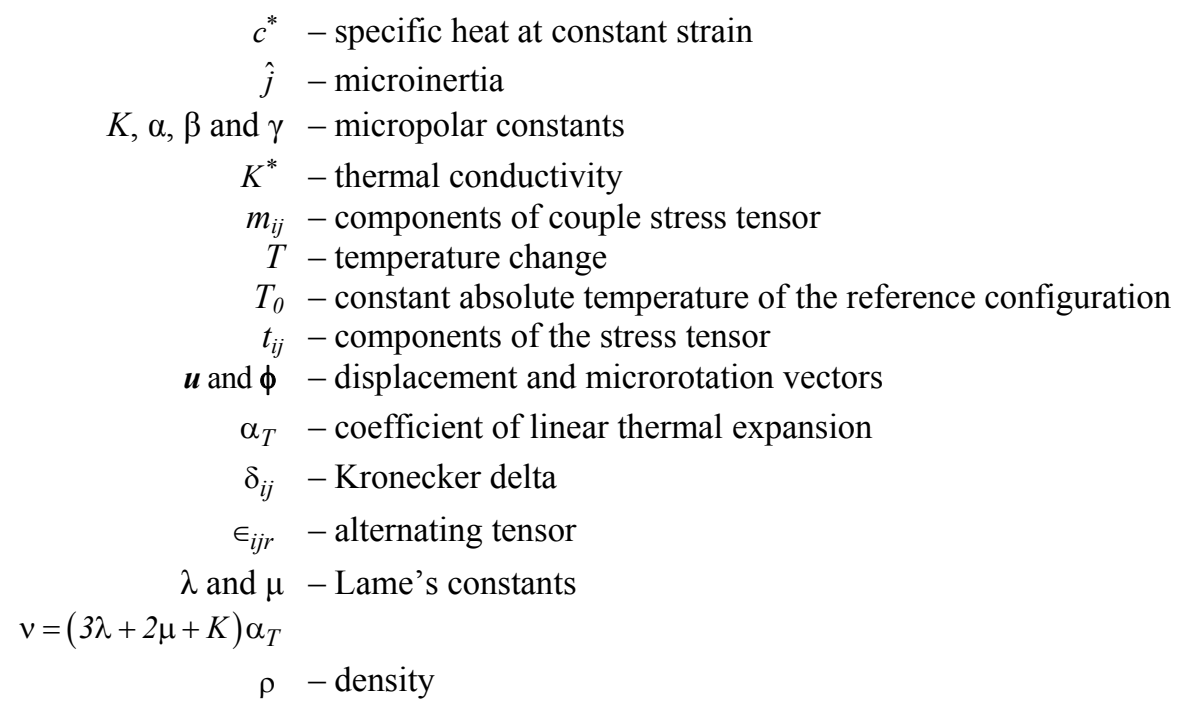

\section{References}

Boley M. (1956): Thermoelastic and irreversible thermodynamics. - Journal of Applied Physics, vol.27, pp.240253.

Boschi E. and Iesan D. (1973): A generalized theory of linear micropolar thermoelasticity. - Meccanica, vol.7, pp.154157.

Chandrasekharaiah D.S. (1986): Heat flux dependent micropolar thermoelasticity. - International Journal of Engineering Science, vol.24, pp.1389-1395.

Chen P.J., Gurtin M.E. and Williams W.O. (1968): A note on non simple heat conduction. - Zeitschrift für angewandte Mathematik und Physik, vol.19, pp.960-970.

Chen P.J., Gurtin M.E. and Williams W.O. (1969): On the thermoelastic material with two temperatures. - Zeitschrift für Angewandte Mathematik und Physik, vol.20, pp.107-112.

Dost S. and Taborrok B. (1978): Generalized micropolar thermoelasticity. - International Journal of Engineering Science, vol.16, pp.173-178.

Eringen A.C. (1966): Linear theory of micropolar elasticity. - Journal of Applied Mathematics and Mechanics, vol.15, pp.909-923.

Eringen A.C. (1970): Foundations of micropolar thermoelasticity. - International Centre for Mechanical Science, Udline Course and Lectures 23, Springen-Verlag, Berlin.

Eringen A.C. (1984): Plane waves in non-local micropolar elasticity. - International Journal of Engineering Science, vol.22, pp.1113-1121. 
Eringen A.C. (1999): Microcontinuum Field Theories I: Foundations and Solids. - Berlin: Springer-Verlag.

Ezzat M.A. and Aiwad E.S. (2010): Constitutive relations, Uniqueness of solution and thermal shock application in the linear theory of micropolar generalized thermoelasticity involving two temperatures. - Journal of Thermal Stresses, vol.33, pp.226-250.

Green A.E. and Lindsay K.A. (1972): Thermoelasticity. - Journal of Elasticity, vol.2, pp.1-7.

Hsia S.Y. and Cheng J.W. (2006): Longitudinal plane waves propagation in elastic micropolar porous media. Japanese Journal of Applied Physics, vol.45, pp.1743-1748.

Hsia S.Y., Chiu S.M., Su C.C. and Chen T.H. (2007): Propagation of transverse waves in elastic micropolar porous semispaces. - Japanese Journal of Applied Physics, vol.46, pp.7399-7405.

Kaushal S., Kumar R. and Miglani A. (2011): Wave propagation in temperature rate dependent thermoelasticity with two temperatures. - Mathematical Sciences, vol.5, pp.125-146.

Kaushal S., Sharma N. and Kumar R. (2010): Propagation of waves in generalized thermoelastic continua with two temperatures. - International Journal of Applied Mechanics and Engineering, vol.15, pp.1111-1127.

Kumar R. (2000): Wave propagation in micropolar viscoelastic generalized thermoelastic solid. - Int. J. Engng. Sci., vol.38, pp.1377-1395.

Kumar R. and Rupender (2008): Reflection at the free surface of magneto-thermo-microstretch elastic solid. - Bull. Pol. Acad. Sci. Tech. Sci., vol.56, pp.263-279.

Kumar R. and Rupender (2009): Propagation of waves in an electro-microstretch generalized thermoelastic semispace. - Acta Mech Sin., vol.25, pp.619-628.

Kumar R. and Sharma J.N. (2005): Reflection of plane waves from the boundaries of a micropolar thermoelastic half space without energy dissipation. - Int. J. Appl. Mech. and Eng., vol.10, pp.631-645.

Kumar R. and Singh B. (1998a): Reflection of plane waves from the flat boundary of a micropolar generalized thermoelastic half space with stretch. - Indian J. Pure Appl. Math., vol.29, pp.657-669.

Magana A. and Quintanilla R. (2008): Uniqueness and growth of solution in two temperature generalized thermoelastic theories. - Maths. Mechs. Solids, Online.

Mukhopadhyay S. and Kumar R. (2009): Thermoelastic interaction on two temperature generalized thermoelasticity in an infinite medium with a cylindrical cavity. - Journal of Thermal Stresses, vol.32, pp.341-360.

Nowacki W. (1986): Theory of Asymmetric Elasticity. - Oxford: Pergamon.

Parfitt V.R. and Eringen A.C. (1969): Reflection of plane waves from the flat boundary of a micropolar elastic halfspace. - J. Acous. Soc. Am., vol.45, pp.1258-1272.

Puri P. and Jordan P. (2006): On the propagation of harmonic plane waves under the two temperature theory. International Journal of Engineering Science, vol.44, pp.1113-1126.

Roushan K. and Santwana M. (2010): Effect of thermal relaxation time on plane wave propagation under two temperature thermoelasticity. - International Journal of Engineering Science, vol.48, pp.128-139.

Singh B. (2007): Wave propagation in an orthotropic micropolar elastic solid. - Int. J. Solid and Struct., vol.44, pp.638-3645.

Singh B. and Kumar R. (1998b): Reflection of plane waves from the flat boundary of a micropolar generalized thermoelastic half space. - Int. J. Engng. Sci., vol.36, pp.865-890.

Tomar S.K., Kumar R. and Kaushik V.P. (1998): Wave propagation of micropolar elastic medium with stretch. - Int. J. Engng. Sci., vol.36, pp.683-698.

Touchert T.R., Claus W.D. Jr. and Ariman T. (1968): The linear theory of micropolar thermoelasticity. - International Journal of Engineering Science 6, pp.37-47. 
Warren W.E. and Chen P.J. (1973): Wave propagation in the two temperature theory of thermoelasticity. - Acta Mechanica, vol.16, pp.21-23.

Youssef H.M. (2005): Theory of two temperature generalized thermoelastic. - IMA Journal of Applied Mathematics, pp.1-8.

Youssef H.M. and Al-Lehaibi E.A. (2007): A state approach of two temperature generalized thermoelasticity of one dimensional problem. - International Journal of Solid and Structures, vol.44, pp.1550-1562.

Youssef H.M. and Al Harby H.A. (2007): State space approach of two temperature generalized thermoelasticity of infinite body with a spherical cavity subjected to different types of thermal loading. - Archive Applied Mechanics, vol.77, pp.675-687.

Received: November 7, 2011

Revised: July 16, 2012 